\title{
REPRESENTATIONS OF ISOTROPIC GAUSSIAN RANDOM FIELDS WITH HOMOGENEOUS INCREMENTS
}

\author{
KACHA DZHAPARIDZE, HARRY VAN ZANTEN, AND PAWEL ZAREBA
}

Received 13 December 2005; Revised 10 May 2006; Accepted 8 June 2006

We present series expansions and moving average representations of isotropic Gaussian random fields with homogeneous increments, making use of concepts of the theory of vibrating strings. We illustrate our results using the example of Lévy's fractional Brownian motion on $\mathbb{R}^{N}$.

Copyright (C) 2006 Kacha Dzhaparidze et al. This is an open access article distributed under the Creative Commons Attribution License, which permits unrestricted use, distribution, and reproduction in any medium, provided the original work is properly cited.

\section{Introduction}

Let $X=(X(t))_{t \in \mathbb{R}^{N}}$ be a zero-mean, mean-square continuous Gaussian random field starting from the origin, that is, $X(0)=0$. Assume that $X$ has homogenous increments, meaning that for every $s \in \mathbb{R}^{N}$, the fields $(X(t)-X(s))_{t \in \mathbb{R}^{N}}$ and $(X(t-s))_{t \in \mathbb{R}^{N}}$ have the same finite-dimensional distributions. Moreover, assume that the field is isotropic, that is, for any $A$ from the group of orthogonal matrices on $\mathbb{R}^{N}$ it holds that $X$ has the same finite-dimensional distributions as the process $(X(A t))_{t \in \mathbb{R}^{N}}$. Under these assumptions we have the spectral representation

$$
\mathbb{E} X(s) X(t)=\int_{\mathbb{R}^{N}}\left(e^{i\langle v, t\rangle}-1\right)\left(e^{-i\langle v, s\rangle}-1\right) d \varrho(v)
$$

for the covariance function of $X$ (see, e.g., [21]). Here $\langle\cdot, \cdot \cdot\rangle$ is the usual inner product on $\mathbb{R}^{N}$, and $\varrho$ is a Borel measure satisfying the condition

$$
\int_{\mathbb{R}^{N}} \frac{\|v\|^{2}}{1+\|v\|^{2}} d \varrho(v)<\infty
$$

In this paper we obtain series expansions and moving average representations for the random field $X$. For any doubly indexed orthonormal basis $S_{l}^{m}$ of the space of square 
integrable functions on the unit sphere $s^{N-1}$ in $\mathbb{R}^{N}$ we can of course write

$$
X(t)=\sum_{l} \sum_{m} S_{l}^{m}\left(\frac{t}{\|t\|}\right) X_{l}^{m}(\|t\|),
$$

where the radial processes $X_{l}^{m}$ are defined by

$$
X_{l}^{m}(r)=\int X(r u) S_{l}^{m}(u) d \sigma_{N}(u)
$$

with $d \sigma_{N}$ the surface area element of $s^{N-1}$. It turns out that if we take for $S_{l}^{m}$ the spherical harmonics (see Section 3), the processes $X_{l}^{m}$ are independent and their distribution only depends on the parameter $l$.

We develop a systematic method to obtain a series expansion and moving average representation for the process $X$ by looking at the radial processes $X_{l}^{m}$ separately. With the spectral measure $\varrho$ on $\mathbb{R}^{N}$ appearing in (1.1) we associate the symmetric Borel measure $\mu$ on the line defined by

$$
\mu(d \lambda)=\frac{\Gamma(N / 2)}{2 \pi^{N / 2}} \lambda^{2} d \Phi(\lambda)
$$

where

$$
\Phi(y)=\int_{\|v\| \leq y} \varrho(d v), \quad y \geq 0 .
$$

Due to (1.2) this measure satisfies the integrability condition

$$
\int \frac{\mu(d \lambda)}{1+\lambda^{2}}<\infty
$$

Next, following the ideas developed in [8], we exploit the fact that a measure of this type can be viewed as the so-called principle spectral measure of a string with a certain mass distribution. Loosely speaking, $\mu$ can be thought of as describing the kinetic energy of a string vibrating at different frequencies (we recall the precise connection in the next section). The general spectral theory of vibrating strings then provides us with the technical tools to obtain the desired representations.

Our representation results apply to general Gaussian isotropic random fields with homogeneous increments. As a consequence of the approach we just outlined, the functions and constants appearing in the theorems are connected to the original process $X$ via the mass distribution associated with the spectral measure $\mu$. Hence, in concrete examples one has to compute the particular mass distribution. In general this is difficult, but there is a number of interesting known cases. In the last section of the paper we highlight the case 
of Lévy's fractional Brownian motion on $\mathbb{R}^{N}$, which is the field with covariance function

$$
\mathbb{E} X(s) X(t)=\frac{1}{2}\left(\|t\|^{2 H}+\|s\|^{2 H}-\|t-s\|^{2 H}\right),
$$

where $H \in(0,1)$ is the so-called Hurst index. For this process the measure $\mu$ has a Lebesgue density equal to a multiple of $\lambda \mapsto|\lambda|^{1-2 H}$. The mass distribution associated with this spectral measure was recently computed in [8]. In combination with our general results this leads to representations of Lévy's fracional Brownian motion extending the one-dimensional results of [7]. We also refer to [16], where closely related results were recently obtained.

The rest of the paper is organized as follows. Section 2 recalls the necessary notions from the spectral theory of vibrating strings. In Section 3 we expand the process $X$ in terms of the spherical harmonics and obtain a first moving average-type result using the vibrating string connection. In Sections 4 and 5 this is further developed into general series and moving average representations. In Section 6 we apply the theory to the particular examples of Lévy's ordinary and fractional Brownian motions.

\section{Introduction to theory of strings}

In this section we present a short account of the spectral theory of vibrating strings. This theory was initiated by M. G. Krein in a series of papers in the 1950s. Here we essentially follow the account given by Dym and McKean [6]. The proofs of all unproved statements in the present section can be found there.

2.1. The vibrating string. A string is described by the pair $\mathbf{l m}$. The number $\mathbf{l} \in(0, \infty]$ is called the length of the string and the nonnegative, right-continuous, nondecreasing function $\mathbf{m}$ defined on the interval $[0,1]$ is called the mass distribution of the string. Values $x \in[0,1]$ are interpreted as locations on the string between the left endpoint $x=0$ and the right endpoint $x=\mathbf{l}$. The value $\mathbf{m}(x)$ is thought of as the total mass of the $[0, x]$-part of the string. The jump of $\mathbf{m}$ at the point $x$ is denoted by $\Delta \mathbf{m}(x)=\mathbf{m}(x)-\mathbf{m}(x-)$. We assume that $\Delta \mathbf{m}(0)=\mathbf{m}(0)$.

It is said that the string is long if $\mathbf{l}+\mathbf{m}(\mathbf{l}-)=\infty$ and short if $\mathbf{l}+\mathbf{m}(\mathbf{l}-)<\infty$. In the case of a short string we need another constant in order to describe the string, that is, the so-called tying constant $\mathbf{k} \in[0, \infty]$. We define also the Hilbert space $L^{2}(\mathbf{m})=L^{2}([0, \mathbf{l}], \mathbf{m})$. The norm on this space is denoted by $\|\cdot\|_{\mathbf{m}}$.

With the general string (not necessarily smooth) we can associate the differential operator

$$
\mathscr{G} f=\frac{d f^{+}}{d \mathbf{m}}
$$

where $f^{+}\left(f^{-}\right)$denotes the right- (left-) hand side derivative of the function $f$. It can be proved (cf. $[6,8])$ that in both cases of long and short strings there exists a dense subset $\mathscr{D}(\mathscr{G})$ of $L^{2}(\mathbf{m})$ such that every $f \in \mathscr{D}(\mathscr{G})$ has left and right derivatives, satisfies $f^{-}(0)=0$ (and $f(\mathbf{l})+\mathbf{k} f^{+}(\mathbf{l})=0$ in case of short string) and the operator $\mathscr{G}: \mathscr{D}(\mathscr{G}) \rightarrow L^{2}(\mathbf{m})$ is well defined, self-adjoint, and negative definite. Let us just remark that the domain $\mathscr{D}(\mathscr{G})$ 
4 Representations of isotropic random fields

consists of functions defined on the real line and satisfying $f(x)=f(0)+x f^{-}(0)$ for $x \leq 0, f(x)=f(\mathbf{l})+(x-\mathbf{l}) f^{+}(\mathbf{l})$ for $x \geq \mathbf{l}$ if $\mathbf{1}<\infty$, and

$$
f(x)=f(0)+f^{-}(0) x+\int_{0}^{x}\left(\int_{[0, y]} \mathscr{G} f(z) d \mathbf{m}(z)\right) d y
$$

for $0 \leq x<1$.

We consider the differential equation $\mathscr{G} A=-\lambda^{2} A$. Since the spectrum of the operator $\mathscr{G}$ is a subset of the half-line $(-\infty, 0]$ (self-adjoint and negative definite), this equation cannot have a solution in $\mathscr{D}(\mathscr{G})$ if $\lambda^{2}$ is not a real, nonnegative number. However, this equation has solutions for any complex $\lambda^{2}$. We define the function $x \mapsto A(x, \lambda)$ as the solution of

$$
\mathscr{G} A(\cdot, \lambda)=-\lambda^{2} A(\cdot, \lambda), \quad A(0, \lambda)=1, \quad A^{-}(0, \lambda)=0 .
$$

The function $A$ can be represented (cf. [6, pages 162 and 171]; [13, page 29]) as follows

$$
A(x, \lambda)=\sum_{n=0}^{\infty}(-1)^{n} \lambda^{2 n} p_{n}(x)
$$

where the $p_{n}$ 's are defined recurrently according to $p_{n}(x)=\int_{0}^{x} \int_{0}^{y} p_{n-1}(z) d \mathbf{m}(z) d y$ and $p_{0}(x)=1$. Thus the function $A(x, \lambda)$ (and $A^{+}(x, \lambda)$ ) for any fixed $x \in[0,1]$ is an entire function of the variable $\lambda$, taking real values for real $\lambda$.

If $\lambda^{2}$ is not a positive real number, we can construct a complementary solution $D(x, \lambda)$ satisfying

$$
\mathscr{G} D(\cdot, \lambda)=-\lambda^{2} D(\cdot, \lambda), \quad D^{-}(0, \lambda)=-1,
$$

by putting

$$
D(x, \lambda)=A(x, \lambda) \int_{x}^{\mathbf{l}+\mathbf{k}} \frac{1}{A^{2}(y, \lambda)} d y .
$$

Another function that will be important in the remainder of this paper is the function

$$
B(x, \lambda)=-\frac{1}{\lambda} A^{+}(x, \lambda)
$$

2.2. Spectral measure of the string. We define the so-called resolvent kernel

$$
r_{\lambda}(x, y)= \begin{cases}A(x, \lambda) D(y, \lambda), & \text { if } x \leq y \\ A(y, \lambda) D(x, \lambda), & \text { if } x \geq y\end{cases}
$$


The name comes from the fact that for any $\lambda^{2}$ outside $[0, \infty)$ we can define the resolvent $R_{\lambda}=\left(-\lambda^{2} I-\mathscr{G}\right)^{-1}$ which can be represented as the integral operator

$$
R_{\lambda} f(x)=\int_{[0, \mathbf{l}]} r_{\lambda}(x, y) f(y) d \mathbf{m}(y) .
$$

Having at hand all required notions, we can now formulate the fundamental theorem.

THeORem 2.1. For every given string, there exists a unique symmetric measure $\mu$ on $\mathbb{R}$ such that

$$
r_{\lambda}(x, y)=\frac{1}{\pi} \int_{\mathbb{R}} \frac{A(x, \omega) A(y, \omega)}{\omega^{2}-\lambda^{2}} \mu(d \omega) .
$$

This measure is called the principal spectral function of the string. Conversely, given a symmetric measure $\mu$ on $\mathbb{R}$ such that

$$
\int_{\mathbb{R}} \frac{\mu(d \lambda)}{1+\lambda^{2}}<\infty
$$

there exists a unique string for which (2.10) holds true.

To make this assertion less abstract, we will now give the reader some idea of the construction of the principal spectral measure. In case of the short string the spectrum of the operator $\mathscr{G}$ is $\left\{-\omega_{n}^{2}: n=1,2, \ldots\right\}$ where $\omega_{n}$ 's are nonnegative roots of the equation

$$
\mathbf{k} A^{+}(\mathbf{l}, \lambda)+A(\mathbf{l}, \lambda)=0
$$

(or $A^{+}(\mathbf{l}, \lambda)=0$ if $\mathbf{k}=\infty$ ). Since $\mathscr{G} A(\cdot, \lambda)=-\lambda^{2} A(\cdot, \lambda)$ for every $\lambda$, the corresponding eigenfunctions are $A\left(\cdot, \omega_{n}\right)$. Now, we define the symmetric measure $\mu$ on the real line which jumps by the amount

$$
\frac{\pi}{2\left\|A\left(\cdot, \omega_{n}\right)\right\|_{\mathbf{m}}^{2}}
$$

at the points $\pm \omega_{n}$. It is not difficult to show that such a measure, indeed, satisfies (2.10) (we use the fact that eigenvalues of the operator $\mathscr{G}$ coincide with eigenvalues of $R_{\lambda}$ which is compact operator on $L^{2}(\mathbf{m})$, hence $A\left(\cdot, \omega_{n}\right)$ form a complete system in which we can expand the resolvent kernel).

If the string is long, we first cut it to make it short. Then construct the measure for the short string according to the procedure described above and let the cutting point tend to infinity.

2.3. The transforms. In this section we introduce the key concept of odd and even transforms. Let $\mu$ be the principal spectral function of the string lmk and let $A$ and $B$ be the functions associated with $\mathbf{m}$. We denote by $L_{\text {even }}^{2}(\mu)$ and $L_{\text {odd }}^{2}(\mu)$ the spaces of all even and, respectively, odd functions in $L^{2}(\mu)$. The norm on $L^{2}(\mu)$ is denoted by $\|\cdot\|_{\mu}$. 
6 Representations of isotropic random fields

Theorem 2.2. The map $\wedge: L^{2}(\mathbf{m}) \rightarrow L_{\text {even }}^{2}(\mu)$ defined by

$$
\wedge: f \longrightarrow \hat{f}_{\text {even }}(\lambda)=\int_{[0,1]} A(x, \lambda) f(x) d \mathbf{m}(x)
$$

is one to one and onto. Its inverse is given by

$$
\vee: \psi \rightarrow \check{\psi}_{\text {even }}(x)=\frac{1}{\pi} \int_{\mathbb{R}} A(x, \lambda) \psi(\lambda) \mu(d \lambda)
$$

It holds that $\left\|\hat{f}_{\mathrm{even}}\right\|_{\mu}^{2}=\pi\|f\|_{\mathbf{m}}^{2}$.

Before introducing the odd analogue of the above, we need to define the space $\mathscr{X}$, which will be the subspace of $L^{2}([0, \mathbf{l}+\mathbf{k}])$ ) (ordinary $L^{2}$-space with respect to Lebesgue measure) of all functions which are constant on a mass-free intervals. Note that $\mathbf{k}=0$ if the string is long. If $\mathbf{k}=\infty$, we require also that the functions vanish on $[1, \infty]$. The ordinary $L^{2}$-norm is denoted by $\|\cdot\|_{2}$.

Theorem 2.3. The map $\wedge: \mathscr{X} \rightarrow L_{\text {odd }}^{2}(\mu)$ defined by

$$
\wedge: f \longrightarrow \hat{f}_{\text {odd }}(\lambda)=\int_{0}^{\mathbf{l}+\mathbf{k}} B(x, \lambda) f(x) d x
$$

is one to one and onto. Its inverse is given by

$$
\vee: \psi \longrightarrow \check{\psi}_{\text {odd }}(x)=\frac{1}{\pi} \int_{\mathbb{R}} B(x, \lambda) \psi(\lambda) \mu(d \lambda)
$$

It holds that $\left\|\hat{f}_{\text {odd }}\right\|_{\mu}^{2}=\pi\|f\|_{2}^{2}$.

Define

$$
T(x)=\int_{0}^{x} \sqrt{\mathbf{m}^{\prime}(y)} d y
$$

where $\mathbf{m}^{\prime}$ is the derivative of the absolute continuous part of $\mathbf{m}$. Let $x(T+)$ and $x(T-)$ denote the biggest and the smallest root $x \in[0,1]$ of the equation

$$
T=\int_{0}^{x} \sqrt{\mathbf{m}^{\prime}(y)} d y
$$

Now we will describe the concept of the Krein space. If $x \in(0, \mathbf{l})$ is a growth point of the string lmk, then we define the class $\mathbf{K}^{x}$ of all functions $f \in L^{2}(\mu)$ that satisfy

$$
\check{f}_{\text {even }}(y)=\check{f}_{\text {odd }}(y)=0 \quad \text { for } y>x \text {. }
$$


Let us introduce one more notion. The entire function $f(z)$ is said to be of exponential type $\tau$ if

$$
\limsup _{R \rightarrow \infty} R^{-1} \max _{|z|=R} \log |f(z)|=\tau
$$

(cf. $[2,6])$.

Denoting by $I^{T}$ the set of all entire functions $f \in L^{2}(\mu)$ of exponential type less than or equal to $T$, we can formulate the following Paley-Wiener-type theorem for this set.

Theorem 2.4. Either $T<T(\mathbf{l})$ and $I^{T}$ coincide with the Krein space $\mathbf{K}^{x(T+)}$ or else $T \geq T(\mathbf{l})$ and $I^{T} \operatorname{span} L^{2}(\mu)$.

In other words, this theorem states that if the function is of finite exponential type, its inverse transforms are supported on a finite interval.

2.4. The orthogonal basis. Let us deal for a while with the short string, assuming $1+$ $\mathbf{m}(\mathbf{l}-)<\infty$ with the tying constant $\mathbf{k}=0$. Consider the family of functions

$$
x \longmapsto A\left(x, \omega_{n}\right), \quad n=1,2, \ldots,
$$

where the $\omega_{n}$ 's are the positive, real zeros of $A(\mathbf{l}, \cdot)$ (we suppress the dependence of $\omega_{n}$ 's on $\mathbf{l}$, but the reader should keep it in mind).

By definition of $A$ and integration by parts we have

$$
\begin{aligned}
-\omega^{2} \int_{0}^{1} A(x, \lambda) A(x, \omega) d \mathbf{m}(x) & =\int_{0}^{1} A(x, \lambda) d A^{+}(x, \omega) \\
& =\left[A(x, \lambda) A^{+}(x, \omega)\right]_{0}^{1}-\int_{0}^{1} A^{+}(x, \omega) A^{+}(x, \lambda) d x .
\end{aligned}
$$

Reversing the roles of $\omega$ and $\lambda$ gives

$$
-\lambda^{2} \int_{0}^{1} A(x, \lambda) A(x, \omega) d \mathbf{m}(x)=\left[A(x, \omega) A^{+}(x, \lambda)\right]_{0}^{1}-\int_{0}^{1} A^{+}(x, \lambda) A^{+}(x, \omega) d x .
$$

Taking the difference of the above two equalities results in

$$
\int_{0}^{1} A(x, \lambda) A(x, \omega) d \mathbf{m}(x)=\frac{A(\mathbf{l}, \omega) A^{+}(\mathbf{l}, \lambda)-A(\mathbf{l}, \lambda) A^{+}(\mathbf{l}, \omega)}{\omega^{2}-\lambda^{2}},
$$

which is the so-called Lagrange identity ([13, Lemma 1.1]; see also [6, page 189, Exercise 3]). Now we easily see that

$$
\int_{0}^{1} A\left(x, \omega_{n}\right) A\left(x, \omega_{k}\right) d \mathbf{m}(x)=\left\|A\left(\cdot, \omega_{n}\right)\right\|_{\mathbf{m}}^{2} \delta_{n}^{k}, \quad k, n=1,2, \ldots,
$$

where $\delta_{n}^{k}$ is Dirac's delta. 
It is also true that the family (2.22) spans the function space $L^{2}(\mathbf{m})$. To show that, let us suppose that there exists $f \in L^{2}(\mathbf{m})$ such that for all $n \in \mathbb{N}$ we have $f \perp A\left(\cdot, \omega_{n}\right)$. It means that

$$
\widehat{f}_{\text {even }}\left(\omega_{n}\right)=\left\langle f, A\left(\cdot, \omega_{n}\right)\right\rangle_{\mathbf{m}}=0, \quad n=1,2, \ldots
$$

Recall that in the present situation the principal spectral measure of the string has atoms only at the points $\pm \omega_{n}$ so that

$$
\int_{\mathbb{R}}\left|\hat{f}_{\text {even }}(\lambda)\right|^{2} \mu(d \lambda)=\sum_{n \in \mathbb{Z}}\left|\hat{f}_{\text {odd }}\left(\omega_{n}\right)\right|^{2} \mu\left(\left\{\omega_{n}\right\}\right)=0 .
$$

According to Theorem 2.2, $\|f\|_{\mathbf{m}}^{2}=1 / \pi\left\|\hat{f}_{\text {even }}\right\|_{\mu}^{2}=0$. Hence, $f=0$ in $L^{2}(\mathbf{m})$. So, we have proved the following.

LEMmA 2.5. If $\mathbf{l}+\mathbf{m}(\mathbf{l}-)<\infty, \mathbf{k}=0$, and $\omega_{n}$ 's $(n=1,2, \ldots)$ are all positive, real zeros of $A(\mathbf{l}, \cdot)$, then the family of functions

$$
\varphi_{n}(x):=\frac{A\left(x, \omega_{n}\right)}{\left\|A\left(\cdot, \omega_{n}\right)\right\|_{\mathbf{m}}}, \quad x \in[0,1], n=1,2, \ldots,
$$

form an orthonormal basis of the function space $L^{2}(\mathbf{m})$.

We would also like to have a basis of the corresponding space $\mathscr{X}$. To achieve this goal we use the Christoffel-Darboux-type relation (cf. [6, Section 6.3, page 234])

$$
\int_{0}^{1} A(x, \omega) A(x, \lambda) d \mathbf{m}(x)+\int_{0}^{1} B(x, \omega) B(x, \lambda) d x=\frac{A(\mathbf{l}, \omega) B(\mathbf{l}, \lambda)-B(\mathbf{l}, \omega) A(\mathbf{l}, \lambda)}{\lambda-\omega} .
$$

Combined with (2.25), it yields the corresponding relation for $B$, that is,

$$
\int_{0}^{1} B(x, \lambda) B(x, \omega) d x=\frac{\omega A(\mathbf{l}, \omega) B(\mathbf{l}, \lambda)-\lambda A(\mathbf{l}, \lambda) B(\mathbf{l}, \omega)}{\lambda^{2}-\omega^{2}} .
$$

Now, we can prove the following.

LEMMA 2.6. If $\mathbf{l}+\mathbf{m}(\mathbf{l}-)<\infty, \mathbf{k}=0$, and $\omega_{n}$ 's $(n=1,2, \ldots)$ are all positive, real zeros of $A(\mathbf{l}, \cdot)$, then the family of functions

$$
\psi_{n}(x):=\frac{B\left(x, \omega_{n}\right)}{\left\|B\left(\cdot, \omega_{n}\right)\right\|_{2}}, \quad x \in[0,1], n=1,2, \ldots,
$$

form an orthonormal basis of the function space $\mathscr{X}$.

Proof. The orthonormality is self-evident by virtue of (2.31). The completeness is shown in the same manner as for (2.22) by using the odd transform instead of even one.

As we will see further on, the norms appearing in the basis functions (2.29) and (2.32) will also appear in the series expansions. Therefore, we will derive a simpler representation of these norms. 
LEMma 2.7. If $\mathbf{1}+\mathbf{m}(\mathbf{l}-)<\infty, \mathbf{k}=0$, and $\omega_{1}<\omega_{2}<\omega_{3}<\cdots$ are positive real zeros of $A(1, \cdot)$, then the norms of the functions $A\left(\cdot, \omega_{n}\right)$ and $B\left(\cdot, \omega_{n}\right)$ in the spaces $L^{2}(\mathbf{m})$ and $L^{2}([0,1])$, respectively, simplify to

$$
\left\|A\left(\cdot, \omega_{n}\right)\right\|_{\mathbf{m}}^{2}=\left\|B\left(\cdot, \omega_{n}\right)\right\|_{2}^{2}=-\left.\frac{1}{2} B\left(\mathbf{l}, \omega_{n}\right) \frac{\partial A(\mathbf{l}, \omega)}{\partial \omega}\right|_{\omega=\omega_{n}} .
$$

Proof. We begin by showing the continuity of the function $A(\cdot, \lambda)$ in the space $L^{2}(\mathbf{m})$ in case of short string, that is, $\mathbf{l}+\mathbf{m}(\mathbf{l}-)<\infty$. In other words, we have to prove that $A(\cdot, \lambda) \rightarrow$ $A(\cdot, \omega)$ in $L^{2}(\mathbf{m})$, as $\lambda \rightarrow \omega$. The mean value theorem ensures existence of such $\gamma_{0}$ between $\lambda$ and $\omega$, that is,

$$
\int_{0}^{1}|A(x, \lambda)-A(x, \omega)|^{2} d \mathbf{m}(x) \leq\left.|\lambda-\omega|^{2} \int_{0}^{1}\left|\frac{\partial A(x, \gamma)}{\partial \gamma}\right|_{\gamma=\gamma_{0}}\right|^{2} d \mathbf{m}(x) .
$$

Using the representation (2.4) of $A(x, \lambda)$ we can establish the upper bound

$$
\left.\int_{0}^{1}\left|\frac{\partial A(x, \gamma)}{\partial \gamma}\right|_{\gamma=\gamma_{0}}\right|^{2} d \mathbf{m}(x) \leq 4 \sum_{n, k \geq 1} n k \gamma_{0}^{2(n+k)-2} \int_{0}^{1} p_{n}(x) p_{k}(x) d \mathbf{m}(x) .
$$

In view of the property $p_{n}(x) \leq(n !)^{-2}[x \mathbf{m}(x)]^{n}$ (see $[6$, page 162$]$ ), we can bound the above integral using

$$
\begin{aligned}
& \sum_{n, k \geq 1} \frac{n k}{(n ! k !)^{2}} \gamma_{0}^{2(n+k)-2} \int_{0}^{\mathbf{l}} x^{n+k} \mathbf{m}(x)^{n+k} d \mathbf{m}(x) \\
& \leq \sum_{n, k \geq 1} \frac{n k}{(n ! k !)^{2}} \gamma_{0}^{2(n+k)-2}(\mathbf{l} \mathbf{m}(\mathbf{l}))^{n+k+1}<\infty,
\end{aligned}
$$

since $\operatorname{lm}(\mathbf{l})<\infty$ by assumption. Hence, we have proved that with some positive finite constant $c$,

$$
\int_{0}^{1}|A(x, \lambda)-A(x, \omega)|^{2} d \mathbf{m}(x) \leq c|\lambda-\omega|^{2}
$$

The same property holds for the function $B(\cdot, \lambda)$. Now, according to formulas $(2.25)$ and (2.31) we can write

$$
\begin{aligned}
\|A(\cdot, \omega)\|_{\mathbf{m}}^{2} & =\lim _{\lambda \rightarrow \omega} \frac{\omega A(\mathbf{l}, \lambda) B(\mathbf{l}, \omega)-\lambda A(\mathbf{l}, \omega) B(\mathbf{l}, \lambda)}{\omega^{2}-\lambda^{2}}, \\
\|B(\cdot, \omega)\|_{2}^{2} & =\lim _{\lambda \rightarrow \omega} \frac{\omega A(\mathbf{l}, \omega) B(\mathbf{l}, \lambda)-\lambda A(\mathbf{l}, \lambda) B(\mathbf{l}, \omega)}{\lambda^{2}-\omega^{2}} .
\end{aligned}
$$


Since both limits are 0/0, application of the l'Hospital's rule (knowing from (2.4) that involved functions are smooth enough) gives us, for $\omega \neq 0$,

$$
\begin{aligned}
\|A(\cdot, \omega)\|_{\mathbf{m}}^{2} & =\frac{\omega[A(\mathbf{l}, \omega)(\partial / \partial \omega) B(\mathbf{l}, \omega)-B(\mathbf{l}, \omega)(\partial / \partial \omega) A(\mathbf{l}, \omega)]+A(\mathbf{l}, \omega) B(\mathbf{l}, \omega)}{2 \omega} \\
\|B(\cdot, \omega)\|_{2}^{2} & =\frac{\omega[A(\mathbf{l}, \omega)(\partial / \partial \omega) B(\mathbf{l}, \omega)-B(\mathbf{l}, \omega)(\partial / \partial \omega) A(\mathbf{l}, \omega)]-A(\mathbf{l}, \omega) B(\mathbf{l}, \omega)}{2 \omega} .
\end{aligned}
$$

Recall $A\left(\mathbf{l}, \omega_{n}\right)=0$ to complete the proof.

So, we have not only found a simple expression for the norms (derivative instead of an integral), but also showed that they are, in fact, the same numbers for $A$ and $B$.

\section{Representations of the covariance}

In this section we present representations of the covariance function of the random field $X$. The results involve the so-called spherical harmonics. These are classical special functions, constituting an orthonormal basis of the space of square integrable functions on the unit sphere in $\mathbb{R}^{N}$. We denote them by $S_{l}^{m}$, with $l=0,1, \ldots$ and $m=1, \ldots, h(l, N)$, where

$$
h(l, N)=\frac{(2 l+N-2)(l+N-3) !}{(N-2) ! l !} .
$$

For details about the spherical harmonics, see, for instance, [9] or [20]. Let us just mention here that the functions can be obtained as eigenfunctions of the Laplace-Beltrami operator on the unit sphere. It holds that each $S_{l}^{m}$ is an eigenfunction corresponding to the eigenvalue $-l(l+N-2)$, and $h(l, N)$ is the dimension of the corresponding eigenspace.

Along with the spherical harmonics, we also make use of the spherical Bessel functions $j_{l}, l=0,1, \ldots$, that are defined in terms of the usual Bessel function of the first kind $J_{\nu}$ of order $v$ as follows:

$$
j_{l}(u)=\Gamma\left(\frac{N}{2}\right) \frac{J_{l+(N-2) / 2}(u)}{(u / 2)^{(N-2) / 2}} .
$$

(We suppress the dependence of the function on $N$ in the notation.) Observe that $j_{l}(0)=$ $\delta_{0}^{l}$. These two sets of spherical functions are related to each other via the Fourier transform: the result known as the Bochner theorem can be found, for example, in [1, Section 9.10]. We will need below only the following partial result:

$$
j_{0}(\lambda\|t\|)=\frac{1}{\left|s^{N-1}(\lambda)\right|} \int_{s^{N-1}(\lambda)} e^{i\langle v, t\rangle} d \sigma_{N}(v)
$$

where $d \sigma_{N}$ is the surface area element of the sphere $s^{N-1}(\lambda)$ with radius $\lambda$ in $\mathbb{R}^{N}$ and

$$
\left|s^{N-1}(\lambda)\right|=\frac{2 \pi^{N / 2}}{\Gamma(N / 2)} \lambda^{N-1}
$$


is its surface area (cf. [20, Section XI.3.2]). In the case of a unit sphere, we will simply write $\left|s^{N-1}(1)\right|=\left|s^{N-1}\right|$. We will also need the so-called addition formula

$$
j_{0}(\lambda\|t-s\|)=\left|s^{N-1}\right| \sum_{l=0}^{\infty} \sum_{m=1}^{h(l, N)} S_{l}^{m}\left(\frac{t}{\|t\|}\right) S_{l}^{m}\left(\frac{s}{\|s\|}\right) j_{l}(\lambda\|t\|) j_{l}(\lambda\|s\|)
$$

(as is given, e.g., by Yaglom [21, page 370] or in [14, page 20]).

For notational convenience we set

$$
G_{l}(r, \lambda)=\frac{j_{l}(0)-j_{l}(r \lambda)}{\lambda}
$$

By using the integral representation of the Bessel function, the so-called Poisson formula as well as it's consequence Gegenbauer's formula (see, e.g., [20, Chapter XI, formulas (3.2.5) and (3.3.7)], resp., or [1, Section 4.7]), we arrive at the following representations:

$$
G_{0}(r, \lambda)=\frac{1}{B(1 / 2,(N-1) / 2)} \int_{-r}^{r}\left(1-\frac{u^{2}}{r^{2}}\right)^{(N-3) / 2} \frac{1-\cos (u \lambda)}{r \lambda} d u
$$

and for $l>0$,

$$
-G_{l}(r, \lambda)=\frac{(-i)^{l-1} B(l, N-1)}{B(1 / 2,(N-1) / 2)} \int_{-r}^{r}\left(1-\frac{u^{2}}{r^{2}}\right)^{(N-1) / 2} C_{l-1}^{N / 2}\left(\frac{u}{r}\right) e^{i \lambda u} d u,
$$

where $C_{l}^{\gamma}$ are the Gegenbauer polynomials. These integral representations show, in particular, that $G_{l}$ 's are alternately odd $(l=0,2, \ldots)$ and even $(l=1,3, \ldots)$ functions of $\lambda$. Moreover, by virtue of the Paley-Wiener theorem (cf. [4] or [6]) we see from (3.7) and from the real and imaginary parts of (3.8) that all the functions $G_{l}(r, \cdot)$ are of exponential type at most $r$. Thus, we have the following.

Lemma 3.1. For each $r \in \mathbb{R}_{+}$, the function $G_{l}(r, \lambda)$ of $\lambda \in \mathbb{R}$ is an odd function for $l=0,2, \ldots$ and an even function for $l=1,3, \ldots$. Moreover, it is an analytic function of finite exponential type less than or equal to $r$.

Our next task is to obtain the representation (3.14) for the covariance function of the random fields $X$. Observe first that due to the homogeneity of the increments, $\mathbb{E} X(s) X(t)$ $=(1 / 2)\left(\mathbb{E}|X(s)|^{2}+\mathbb{E}|X(t)|^{2}-\mathbb{E}|X(t-s)|^{2}\right)$. Since, in addition, our field is isotropic, the variance $\mathbb{E}|X(t)|^{2}$ is a function only of the norm of $t$. Denoting this function (called by Yaglom [21] the structure function) by $D$ we thus write $D(\|t\|)=\mathbb{E}|X(t)|^{2}$. With this notation the covariance can be rewritten as

$$
\mathbb{E} X(s) X(t)=\frac{1}{2}(D(\|s\|)+D(\|t\|)-D(\|t-s\|)) .
$$

By putting $t=s$ in (1.1), we get the following spectral representation for the structure function:

$$
D(\|t\|)=2 \int_{\mathbb{R}^{N}}\left(1-e^{i\langle v, t\rangle}\right) \varrho(d v)=2 \int_{\mathbb{R}^{N}}(1-\cos \langle v, t\rangle) \varrho(d v)
$$

(the imaginary part vanishes, since our field $X$ is real, cf. [21, page 435]). 
It is useful to associate with the spectral measure $\varrho$ the bounded nondecreasing function $\Phi$ defined by (1.6). Note that condition (1.2) implies

$$
\int_{0}^{\infty} \frac{\lambda^{2}}{1+\lambda^{2}} d \Phi(\lambda)<\infty
$$

By rewriting the variable $v=\left(v_{1}, \ldots, v_{N}\right)$ in polar coordinates with radius $\lambda=\|v\|$, we get $\left|s^{N-1}(\lambda)\right| \varrho(d v)=d \sigma_{N}(v) d \Phi(\lambda)$ (cf. (3.3) and (3.4)). Due to formula (3.3), the representation (3.10) can be rewritten in polar coordinates as

$$
D(r)=2 \int_{0}^{\infty}\left(1-j_{0}(r \lambda)\right) d \Phi(\lambda)
$$

Formula (3.9) for the covariance function then becomes

$$
\mathbb{E} X(s) X(t)=\int_{0}^{\infty}\left[1-j_{0}(\lambda\|t\|)-j_{0}(\lambda\|s\|)+j_{0}(\lambda\|t-s\|)\right] d \Phi(\lambda)
$$

The following representation of the covariance function is implicit in [16]. Since it serves as starting point in our considerations, we provide an explicit proof.

Theorem 3.2. The covariance function of the isotropic Gaussian random field X with homogeneous increments can be represented as follows:

$$
\begin{aligned}
\mathbb{E} X(s) X(t)= & \left|s^{N-1}\right| \sum_{l=0}^{\infty} \sum_{m=1}^{h(l, N)} S_{l}^{m}\left(\frac{t}{\|t\|}\right) S_{l}^{m}\left(\frac{s}{\|s\|}\right) \\
& \times \int_{0}^{\infty} G_{l}(\|t\|, \lambda) G_{l}(\|s\|, \lambda) \lambda^{2} d \Phi(\lambda) .
\end{aligned}
$$

Proof. Note that $h(0, N)=1, S_{0}^{1}(\cdot)$ is a constant function for every $N$ and since the spherical harmonics are orthonormal, this constant is given by $S_{0}^{1}(\cdot) \equiv 1 / \sqrt{\left|s^{N-1}\right|}$. Hence, (3.14) is equivalent to

$$
\begin{aligned}
\mathbb{E} X(s) X(t)-\int_{0}^{\infty}\left(1-j_{0}(\lambda\|t\|)\right)\left(1-j_{0}(\lambda\|s\|)\right) d \Phi(\lambda) \\
=\left|s^{N-1}\right| \sum_{l=1}^{\infty} \sum_{m=1}^{h(l, N)} S_{l}^{m}\left(\frac{t}{\|t\|}\right) S_{l}^{m}\left(\frac{s}{\|s\|}\right) \int_{0}^{\infty} j_{l}(\lambda\|t\|) j_{l}(\lambda\|s\|) d \Phi(\lambda)
\end{aligned}
$$

which we are now going to prove. The addition formula (3.5) implies

$$
\begin{aligned}
& j_{0}(\lambda\|t-s\|)-j_{0}(\lambda\|t\|) j_{0}(\lambda\|s\|) \\
& \quad=\left|s^{N-1}\right| \sum_{l=1}^{\infty} \sum_{m=1}^{h(l, N)} S_{l}^{m}\left(\frac{t}{\|t\|}\right) S_{l}^{m}\left(\frac{s}{\|s\|}\right) j_{l}(\lambda\|t\|) j_{l}(\lambda\|s\|) .
\end{aligned}
$$


Taking the integral with respect to $d \Phi(\lambda)$ on both sides we see that the expression on the right-hand side of (3.15) is equal to the integral

$$
\int_{0}^{\infty}\left(j_{0}(\lambda\|t-s\|)-j_{0}(\lambda\|t\|) j_{0}(\lambda\|s\|)\right) d \Phi(\lambda)
$$

But in view of (3.13) we see that also the left-hand side of (3.15) equals to the latter integral. Thus (3.15) holds true.

We now introduce the spectral measure $\mu$ defined by

$$
\mu(d \lambda)=\frac{\lambda^{2} d \Phi(\lambda)}{\left|s^{N-1}\right|}
$$

and view it as the principle spectral measure of a unique string lmk in the sense of Theorem 2.1. Note that condition (2.11) is ensured due to (3.11).

Corollary 3.3. The covariance function of the isotropic Gaussian random field $X$ with homogeneous increments can be represented as follows:

$$
\begin{aligned}
\mathbb{E} X(s) X(t)= & \pi\left|s^{N-1}\right|^{2} \sum_{l=0,2, \ldots} \sum_{m=1}^{h(l, N)} S_{l}^{m}\left(\frac{t}{\|t\|}\right) S_{l}^{m}\left(\frac{s}{\|s\|}\right) \int_{0}^{1+\mathbf{k}} \check{G}_{l}(\|t\|, x) \check{G}_{l}(\|s\|, x) d x \\
& +\pi\left|s^{N-1}\right|^{2} \sum_{l=1,3, \ldots} \sum_{m=1}^{h(l, N)} S_{l}^{m}\left(\frac{t}{\|t\|}\right) S_{l}^{m}\left(\frac{s}{\|s\|}\right) \int_{0}^{1} \check{G}_{l}(\|t\|, x) \check{G}_{l}(\|s\|, x) d \mathbf{m}(x),
\end{aligned}
$$

where

$$
\begin{array}{ll}
\check{G}_{l}(r, x)=\frac{1}{\pi} \int_{\mathbb{R}} G_{l}(r, \lambda) A(x, \lambda) \mu(d \lambda), & l=1,3, \ldots, \\
\check{G}_{l}(r, x)=\frac{1}{\pi} \int_{\mathbb{R}} G_{l}(r, \lambda) B(x, \lambda) \mu(d \lambda), & l=0,2, \ldots,
\end{array}
$$

and the functions $A(x, \lambda)$ and $B(x, \lambda)$ are the eigenfunctions associated with the string $\mathbf{l m k}$ whose principal spectral measure $\mu$ is given by (3.18).

Proof. Condition (2.11) ensures that the measure $\mu$ satisfies the assumptions of Theorem 2.1. By virtue of this theorem there exists an unique associated string with mass $\mathbf{m}$ and length $\mathbf{l} \leq \infty$. Note that the function $\breve{G}_{l}(r, x)$ is defined as the even or odd (for appropriate $l$ 's) inverse transform of the function $G_{l}(r, \lambda)$. Since transforms are isometries, we have

$$
\begin{array}{ll}
\left\langle G_{l}\left(r_{1}, \cdot\right), G_{l}\left(r_{2}, \cdot\right)\right\rangle_{\mu}=\pi\left\langle\check{G}_{l}\left(r_{1}, \cdot\right), \check{G}_{l}\left(r_{2}, \cdot\right)\right\rangle_{\mathrm{m}}, & l=1,3, \ldots, \\
\left\langle G_{l}\left(r_{1}, \cdot\right), G_{l}\left(r_{2}, \cdot\right)\right\rangle_{\mu}=\pi\left\langle\check{G}_{l}\left(r_{1}, \cdot\right), \check{G}_{l}\left(r_{2}, \cdot\right)\right\rangle_{2}, & l=0,2, \ldots
\end{array}
$$

The proof is completed by applying this to representation (3.14). 
Remark 3.4. Recall the assertion of Lemma 3.1 that the function $G_{l}(r, \cdot)$ is of finite exponential type at most $r$. Combined with Theorem 2.4, this implies that the inverse transforms of such functions are supported on the finite interval $[0, x(r+)]$ and that the representation (3.19) is in fact of the form

$$
\begin{aligned}
\mathbb{E} X(s) X(t)= & \pi\left|s^{N-1}\right|^{2} \sum_{l=0,2, \ldots} \sum_{m=1}^{h(l, N)} S_{l}^{m}\left(\frac{t}{\|t\|}\right) S_{l}^{m}\left(\frac{s}{\|s\|}\right) \int_{0}^{n(s, t)} \check{G}_{l}(\|t\|, y) \check{G}_{l}(\|s\|, y) d y \\
& +\pi\left|s^{N-1}\right|^{2} \sum_{l=1,3, \ldots} \sum_{m=1}^{h(l, N)} S_{l}^{m}\left(\frac{t}{\|t\|}\right) S_{l}^{m}\left(\frac{s}{\|s\|}\right) \int_{0}^{n(s, t)} \check{G}_{l}(\|t\|, y) \check{G}_{l}(\|s\|, y) d \mathbf{m}(y)
\end{aligned}
$$

with $n(s, t):=x(\|t\|+) \wedge x(\|s\|+)$. This immediately allows us to write down the following moving average-type representation of the random field $X$ :

$$
X(t)=\sqrt{\pi}\left|s^{N-1}\right| \sum_{l=0}^{\infty} \sum_{m=1}^{h(l, N)} S_{l}^{m}\left(\frac{t}{\|t\|}\right) \int_{0}^{x(\|t\|+)} \check{G}_{l}(\|t\|, y) d M_{l}^{m}(y),
$$

where for $l=0,1, \ldots$ the sets $\left\{M_{l}^{m}, m=1, \ldots, h(l, N)\right\}$ consist of $h(l, N)$ independent copies of mutually independent Gaussian processes $M_{l}$ with independent increments, whose variances are given by

$$
\mathbb{E}\left|M_{l}(y)\right|^{2}= \begin{cases}y, & l=0,2, \ldots \\ \mathbf{m}(y), & l=1,3, \ldots\end{cases}
$$

In section 5 we will return to this subject.

\section{Series expansion}

In this section we restrict the parameter $t$ to the ball of radius $T$, that is,

$$
t \in \mathscr{B}_{T}:=\left\{u \in \mathbb{R}^{N}:\|u\| \leq T\right\}
$$

We consider a string with the same mass function $\mathbf{m}$ (associated via Theorem 2.1 with $\mu$ defined by (3.18)) but we cut it at the point $\mathbf{l}:=x(T+)$ (which we assume to be finite) with tying constant $\mathbf{k}=0$ and $\mathbf{m}(\mathbf{l}-)<\infty$.

Let us concentrate for a moment on the odd $l$ 's. Since $\check{G}_{l}(\|t\|, \cdot)$ then belongs to the space $L^{2}(\mathbf{m})$, we can expand it in basis (2.29) so that

$$
\check{G}_{l}(\|t\|, x)=\sum_{n=0}^{\infty}\left\langle\check{G}_{l}(\|t\|, \cdot), \varphi_{n}\right\rangle_{\mathbf{m}} \varphi_{n}(x) .
$$


Having this, we can write

$$
\begin{aligned}
& \int_{0}^{1} \check{G}_{l}(\|t\|, x) \check{G}_{l}(\|s\|, x) d \mathbf{m}(x) \\
& \quad=\sum_{n=0}^{\infty}\left(\int_{0}^{1} \check{G}_{l}(\|t\|, x) \varphi_{n}(x) d \mathbf{m}(x)\right)\left(\int_{0}^{1} \check{G}_{l}(\|s\|, x) \varphi_{n}(x) d \mathbf{m}(x)\right),
\end{aligned}
$$

which is the same as

$$
\int_{0}^{1} \check{G}_{l}(\|t\|, x) \check{G}_{l}(\|s\|, x) d \mathbf{m}(x)=\sum_{n=0}^{\infty} \frac{G_{l}\left(\|t\|, \omega_{n}\right) G_{l}\left(\|s\|, \omega_{n}\right)}{\left\|A\left(\cdot, \omega_{n}\right)\right\|_{\mathbf{m}}^{2}},
$$

since

$$
\int_{0}^{1} \check{G}_{l}(\|t\|, x) \varphi_{n}(x) d \mathbf{m}(x)=\frac{G_{l}\left(\|t\|, \omega_{n}\right)}{\left\|A\left(\cdot, \omega_{n}\right)\right\|_{\mathbf{m}}} .
$$

Exactly the same argument for even l's results in corresponding formula

$$
\int_{0}^{1} \check{G}_{l}(\|t\|, x) \check{G}_{l}(\|s\|, x) d x=\sum_{n=0}^{\infty} \frac{G_{l}\left(\|t\|, \omega_{n}\right) G_{l}\left(\|s\|, \omega_{n}\right)}{\left\|B\left(\cdot, \omega_{n}\right)\right\|_{2}^{2}} .
$$

Then, keeping in mind Lemma 2.7, we can rewrite representation (3.19) as follows:

$$
\mathbb{E} X(s) X(t)=\pi\left|s^{N-1}\right|^{2} \sum_{l=0}^{\infty} \sum_{m=1}^{h(l, N)} S_{l}^{m}\left(\frac{t}{\|t\|}\right) S_{l}^{m}\left(\frac{s}{\|s\|}\right) \sum_{n=0}^{\infty} \frac{G_{l}\left(\|t\|, \omega_{n}\right) G_{l}\left(\|s\|, \omega_{n}\right)}{\left\|A\left(\cdot, \omega_{n}\right)\right\|_{\mathbf{m}}^{2}} .
$$

Now we can prove the following.

Theorem 4.1. Let $X$ be a centered, mean-square continuous Gaussian isotropic random field with homogenous increments on $\mathbb{R}^{N}$. If the mass function associated with $\mu$ (cf. (3.18)) is such that $x(T+)+\mathbf{m}(x(T+)-)<\infty$ for $T>0$, then the following representation holds:

$$
X(t)=\sum_{n=0}^{\infty} \sum_{l=0}^{\infty} \sum_{m=1}^{h(l, N)} S_{l}^{m}\left(\frac{t}{\|t\|}\right) G_{l}\left(\|t\|, \omega_{n}\right) \xi_{l, n}^{m}, \quad t \in \mathscr{S}_{T}
$$

where $\xi_{l, n}^{m}$ are independent, mean-zero Gaussian random variables with variances

$$
\sigma_{n}^{2}=-\frac{2 \pi\left|s^{N-1}\right|^{2}}{\left.B\left(x(T+), \omega_{n}\right)(\partial / \partial \omega) A(x(T+), \omega)\right|_{\omega=\omega_{n}}}
$$

and the $\omega_{n}$ 's are the zeros of $A(x(T+), \cdot)$. This series converges in mean-square sense for any fixed $t \in \mathscr{B}_{T}$. Moreover, if the process $(X(t))_{\|t\|<T}$ is continuous, the series converges with probability one in the space of continuous functions $C\left(\mathscr{S}_{T}\right)$ endowed with the supremum norm. 
Proof. For $M \in \mathbb{N}$, consider the partial sum of the series defined by

$$
X^{M}(t)=\sum_{n, l=0}^{M} \sum_{m=1}^{h(l, N)} S_{l}^{m}\left(\frac{t}{\|t\|}\right) G_{l}\left(\|t\|, \omega_{n}\right) \xi_{l, n}^{m}
$$

The covariance representation (4.7) ensures, as $M \rightarrow \infty$, mean-square convergence of $X^{M}(t)$ to process $X(t)$ for every $t$.

For the remainder of the proof, assume that $X$ is continuous. The pointwise meansquare convergence implies weak convergence of the finite-dimensional distributions. So if we manage to prove the asymptotic tightness in $C(\mathscr{B})$ of the sequence $X^{M}$, we are able to use [19, Theorem 1.5.4] which states that weak convergence of finite-dimensional distributions combined with asymptotic tightness is sufficient for the sequence to converge weakly in $C\left(\mathscr{B}_{T}\right)$. By virtue of the Itô-Nisio theorem (see, e.g., [19]) this is equivalent to convergence with probability one in $C\left(\mathscr{B}_{T}\right)$. Now we will prove the asymptotic tightness of $X^{M}$ in the space $C\left(\mathscr{B}_{T}\right)$.

Asymptotic tightness is equivalent (see, e.g., [19, Theorem 1.5.7]) to the following two conditions:

(i) $X^{M}(t)$ is asymptotically tight in $\mathbb{R}$ for every fixed $t \in \mathscr{B}_{T}$;

(ii) there exists semimetric $d$ on $\mathscr{B}_{T}$ such that $\left(\mathscr{B}_{T}, d\right)$ is totally bounded and $\left(X^{M}(t)\right)_{\|t\|<T}$ is asymptotically uniformly $d$-equicontinuous in probability, that is, for all $\varepsilon, \eta>0, \exists \delta>0$ such that

$$
\limsup _{M \rightarrow \infty} \mathbb{P}\left(\sup _{d(s, t)<\delta}\left|X^{M}(t)-X^{M}(s)\right|>\varepsilon\right)<\eta .
$$

The first condition is automatically satisfied by virtue of the weak convergence of the partial sums for every $t$. It suffices to prove the second one.

Let us define a sequence of semimetrics on $\mathscr{B}_{T}$ by

$$
d_{M}^{2}(s, t):=\mathbb{E}\left|X^{M}(t)-X^{M}(s)\right|^{2} \leq \mathbb{E}|X(t)-X(s)|^{2}=: d^{2}(s, t) .
$$

It is known (see, e.g., [19, page 446]) that for any $M$, any Borel probability measure $v$ on $\left(\mathscr{B}_{T}, d_{M}\right)$, and every $\delta, \eta>0$ it holds that

$$
\mathbb{E} \sup _{d_{M}(s, t)<\delta}\left|X^{M}(t)-X^{M}(s)\right| \lesssim \sup _{t} \int_{0}^{\eta} \sqrt{\log \frac{1}{\nu\left(\mathscr{B}_{\varepsilon}\left(t, d_{M}\right)\right)}} d \varepsilon+\delta \sqrt{N\left(\eta, \mathscr{P}_{T}, d_{M}\right)},
$$

where $\mathscr{B}_{\varepsilon}(t, d)$ denotes the ball of radius $\varepsilon$ around point $t$ in metric $d$ and $N(\eta, \mathbf{Y}, d)$ is so-called $\eta$-covering number, that is, the minimal number of balls of radius $\eta$ needed to cover $\mathbf{Y}$. Since $d_{M}(s, t) \leq d(s, t)$, we have

$$
\mathbb{E} \sup _{d(s, t)<\delta}\left|X^{M}(t)-X^{M}(s)\right| \leq \mathbb{E} \sup _{d_{M}(s, t)<\delta}\left|X^{M}(t)-X^{M}(s)\right| .
$$

Proposition A.2.17 of [19] applied to the process $X$ itself (uniform continuity and boundness with respect to standard deviation semimetric $d(s, t)$ is equivalent to the continuity 
of almost all sample paths with respect to Euclidean distance and continuity of the map $t \rightarrow \mathbb{E}|X(t)|^{2}$ (cf. [19, Lemma 1.5.9]), the latter being satisfied by virtue of the meansquare continuity) yields that there exists some Borel probability measure $v^{*}$ on $\left(\mathscr{B}_{T}, d\right)$ such that

$$
\sup _{t \in \mathscr{\Re}_{T}} \int_{0}^{\eta} \sqrt{\log \frac{1}{\nu^{*}\left(\mathscr{S}_{\varepsilon}(t, d)\right)}} d \varepsilon \stackrel{\eta\urcorner 0}{\longrightarrow} 0 .
$$

From relation $d_{M} \leq d$ we can easily see that $d_{M}$-open sets are also $d$-open sets. It implies that $\sigma$-algebras of Borel sets satisfy $\mathscr{B}\left(\mathscr{B}_{T}, d_{M}\right) \subset \mathscr{B}\left(\mathscr{B}_{T}, d\right)$. Hence, the measure $v^{*}$ is also a Borel measure on $\left(\mathscr{B}_{T}, d_{M}\right)$. By choosing (4.13) for the measure $v^{*}$ and combining it with (4.14), we get

$$
\begin{aligned}
\mathbb{E} \sup _{d(s, t)<\delta}\left|X^{M}(t)-X^{M}(s)\right| & \lesssim \sup _{t \in \mathscr{B}_{T}} \int_{0}^{\eta} \sqrt{\log \frac{1}{v^{*}\left(\mathscr{S}_{\varepsilon}\left(t, d_{M}\right)\right)}} d \varepsilon+\delta \sqrt{N\left(\eta, \mathscr{B}_{T}, d_{M}\right)} \\
& \leq \sup _{t \in \mathscr{B}_{T}} \int_{0}^{\eta} \sqrt{\log \frac{1}{v^{*}\left(\mathscr{S}_{\varepsilon}(t, d)\right)}} d \varepsilon+\delta \sqrt{N\left(\eta, \mathscr{S}_{T}, d\right) .}
\end{aligned}
$$

The first term on the right-hand side can be made arbitrarily small by (4.15). It is not difficult to see that condition (4.15) is sufficient for the space $\left(\mathscr{B}_{T}, d\right)$ to be totally bounded (see, e.g., [19, page 446]). Hence, the number $N\left(\eta, \mathscr{S}_{T}, d\right)$ is finite and also the second term on the right-hand side can be arbitrarily small. This proves the desired equicontinuity of $X^{M}$.

Remark 4.2. Notice that our expansion (4.8) is of a different form than the one derived by Malyarenko [16, Theorem 1]. The conditions of the latter theorem seem difficult to verify, except in the case of Lévy's fractional Brownian motion.

\section{Moving average for smooth strings}

In this section we will show how the representation (3.23) simplifies when the string associated to the random field has a smooth mass function. We obtain an integral representation in the time domain, which can be viewed as a multivariate moving average representation.

To this end, we have to invert the function $t(x)=\int_{0}^{x} \sqrt{\mathbf{m}^{\prime}(y)} d y$ defined in Section 2. Therefore, we need to require that the mass function is continuously differentiable with a positive derivative. This then yields the following representation of the covariance function in the time domain.

THeOREM 5.1. If the mass function $\mathbf{m}$ associated with the random field $X$ is continuously differentiable and $\mathbf{m}^{\prime}>0$, then for every $s, t \in \mathbb{R}^{N}$,

$$
\begin{aligned}
\mathbb{E} X(s) X(t)= & 2 \pi^{2}\left|s^{N-1}\right|^{2} \sum_{l=0}^{\infty} \sum_{m=1}^{h(l, N)} S_{l}^{m}\left(\frac{t}{\|t\|}\right) S_{l}^{m}\left(\frac{s}{\|s\|}\right) \\
& \times \int_{0}^{\|s\| \wedge\|t\|} k_{l}(\|t\|, u) k_{l}(\|s\|, u) d V(2 u),
\end{aligned}
$$


18 Representations of isotropic random fields

where $V(2 u)=\pi^{-1} \mathbf{m}(x(u))$ and the kernels are given by

$$
\begin{gathered}
k_{l}(\|t\|, u)=\check{G}_{l}(\|t\|, x(u)) x^{\prime}(u), \quad l=0,2, \ldots, \\
k_{l}(\|t\|, u)=\check{G}_{l}(\|t\|, x(u)), \quad l=1,3, \ldots,
\end{gathered}
$$

for $u \leq\|t\|$.

Proof. Let us first derive some useful relations between the functions $\mathbf{m}, x$, and $V$. Differentiating $t=\int_{0}^{x(t)} \sqrt{\mathbf{m}^{\prime}(y)} d y$ we obtain

$$
x^{\prime}(t)=\frac{1}{\sqrt{\mathbf{m}^{\prime}(x(t))}} .
$$

Since

$$
\mathbf{m}^{\prime}(x(t)) x^{\prime}(t)=2 \pi V^{\prime}(2 t)
$$

from (5.3) we get

$$
2 \pi V^{\prime}(2 t) x^{\prime}(t)=1 .
$$

To prove the representation (5.1) we apply the change of variable $y=x(u)$ to both terms on the right-hand side of (3.22). Due to (5.5), the measure $d y$ in the integral of the first term becomes

$$
x^{\prime}(u) d u=2 \pi x^{\prime}(u)^{2} d V(2 u)
$$

Hence,

$$
\begin{aligned}
& \int_{0}^{n(s, t)} \check{G}_{l}(\|t\|, y) \check{G}_{l}(\|s\|, y) d y \\
& \quad=2 \pi \int_{0}^{\|s\| \wedge\|t\|} \check{G}_{l}(\|t\|, x(u)) \check{G}_{l}(\|s\|, x(u)) x^{\prime}(u)^{2} d V(2 u) .
\end{aligned}
$$

The same change of variables allows us to write the integral of the second term in (3.22) in the following manner:

$$
\begin{aligned}
\int_{0}^{n(s, t)} \check{G}_{l}(\|t\|, y) \check{G}_{l}(\|s\|, y) d \mathbf{m}(y) \\
\quad=2 \pi \int_{0}^{\|s\| \wedge\|t\|} \check{G}_{l}(\|t\|, x(u)) \check{G}_{l}(\|s\|, x(u)) d V(2 u),
\end{aligned}
$$

since the measure $d \mathbf{m}(y)=\mathbf{m}^{\prime}(y) d y$ turns into $\mathbf{m}^{\prime}(x(u)) x^{\prime}(u) d u=2 \pi d V(2 u)$, (cf. (5.4)). Due to (5.7) and (5.8) the representation (3.22) turns into (5.1).

COROLlary 5.2. Under assumptions of Theorem 5.1,

$$
X(t)=\sqrt{2} \pi\left|s^{N-1}\right| \sum_{l=0}^{\infty} \sum_{m=1}^{h(l, N)} S_{l}^{m}\left(\frac{t}{\|t\|}\right) \int_{0}^{\|t\|} k_{l}(\|t\|, u) d M_{l}^{m}(u)
$$


where $\left\{M_{l}^{m}\right\}$ are independent copies of the Gaussian martingale $M$ with zero mean and variance function $E|M(u)|^{2}=V(2 u)$.

Remark 5.3. The representation (5.1) may be compared with a similar result by Malyarenko [16] that is derived under a number of conditions on the spectral measure, listed in his [16, Theorem 1].

\section{Examples}

This section is devoted to applications of our general results first to Lévy's Brownian motion and then to Lévy's fractional Brownian motion of arbitrary Hurst index.

6.1. Lévy's Brownian motion. Lévy [15] defined the Brownian motion on $\mathbb{R}^{N}$ as a centered Gaussian random field with a covariance structure

$$
\mathbb{E} X(t) X(s)=\frac{1}{2}(\|t\|+\|s\|-\|t-s\|) .
$$

Properties of this field were investigated by several authors, see, for instance, Chentsov [3], McKean Jr. [17], and Molchan [18]. Since the structure function in this case is simply $D(r)=r$, we can easily verify via formula (3.12) that the corresponding spectral measure is given by $\lambda^{2} \Phi^{\prime}(\lambda)=\left|s^{N-1}\right| /\left|s^{N}\right|$. To see this rewrite (3.12) in the form

$$
r=-2 \int_{0}^{r} d u \int_{0}^{\infty} j_{0}^{\prime}(u \lambda) d \Phi(\lambda)=\frac{\left|s^{N-1}\right|}{\left|s^{N}\right|} 2^{N / 2} \Gamma\left(\frac{N}{2}\right) \int_{0}^{r} d u \int_{0}^{\infty} \frac{J_{N / 2}(z)}{z^{N / 2}} d z
$$

and apply [12, formula (6.561.14)] to evaluate the last integral.

Thus by (3.18) we have $\mu(d \lambda)=d \lambda /\left|s^{N}\right|$. It is now easy to determine the corresponding mass function of the string. As we know (cf. $[6,8]$ ) the mass function associated with the Lebesgue spectral measure is $\mathbf{m}(x)=x$. In order to handle the constant multiplier that presently occurs, only "rule 1" of [6, page 265], is required. It tells us that the multiplication of a spectral measure by constant $c$ changes the corresponding mass function to $c^{-1} \mathbf{m}\left(c^{-1} x\right)$ and the eigenfunctions $A$ and $B$ to $A\left(c^{-1} x, \lambda\right)$ and $c^{-1} B\left(c^{-1} x, \lambda\right)$. Hence the mass function associated with Lévy's Brownian motion is $\mathbf{m}(x)=\left|s^{N}\right|{ }^{2} x$, while $A(x, \lambda)=\cos \left(\left|s^{N}\right| \lambda x\right)$ and $B(x, \lambda)=\left|s^{N}\right| \sin \left(\left|s^{N}\right| \lambda x\right)$. But since in this case $x(t)=t /\left|s^{N}\right|$, the constant disappears:

$$
V(2 t)=\frac{m(x(t))}{\pi}=\frac{t}{\pi}, \quad A(x(t), \lambda)=\cos (\lambda t), \quad B(x(t), \lambda) x^{\prime}(t)=\sin (\lambda t) .
$$

In this case the transforms in Section 2.3 are the Fourier cosine and sine transforms. Equations (3.20), in conjuncture with (5.2), become

$$
\begin{gathered}
k_{2 n+1}(r, u)=\check{G}_{2 n+1}(r, x(u))=\frac{2}{\pi\left|s^{N}\right|} \int_{0}^{\infty} G_{2 n+1}(r, \lambda) \cos (u \lambda) d \lambda, \\
k_{2 n}(r, u)=\check{G}_{2 n}(r, x(u)) x^{\prime}(u)=\frac{2}{\pi\left|s^{N}\right|} \int_{0}^{\infty} G_{2 n}(r, \lambda) \sin (u \lambda) d \lambda
\end{gathered}
$$


for $n=0,1,2, \ldots$. By definition (3.6), we deal here with the cosine transform of the function $J_{2 n+N / 2}(r \lambda) / \lambda^{N / 2}$ and for $n>0$ with the sine transform of the function $J_{2 n-1+N / 2}(r \lambda) /$ $\lambda^{N / 2}$ to be found in the tables in [10]; see formulas (1.12.10) or (1.12.13) for the cosine transform and formulas $(2.12 .10)$ or $(2.12 .11)$ for the sine transform. We get, with $F$ denoting the Gauss hypergeometric function,

$$
\begin{aligned}
\pi\left|s^{N-1}\right| k_{2 n+1}(r, u) & =\frac{(-1)^{n} \Gamma(N) \Gamma(2 n+1)}{\Gamma(2 n+N)}\left(1-\frac{u^{2}}{r^{2}}\right)^{(N-1) / 2} C_{2 n}^{N / 2}\left(\frac{u}{r}\right) \\
& =-\frac{\Gamma((N+1) / 2) \Gamma(n+1 / 2)}{\sqrt{\pi} \Gamma(n+N / 2)}\left(1-\frac{u^{2}}{r^{2}}\right)^{(N-1) / 2} F\left(-n, n+\frac{N}{2} ; \frac{1}{2} ; \frac{u^{2}}{r^{2}}\right),
\end{aligned}
$$

and for $n>0$,

$$
\begin{aligned}
\pi\left|s^{N-1}\right| k_{2 n}(r, u) & =\frac{(-1)^{n} \Gamma(N) \Gamma(2 n)}{\Gamma(2 n-1+N)}\left(1-\frac{u^{2}}{r^{2}}\right)^{(N-1) / 2} C_{2 n-1}^{N / 2}\left(\frac{u}{r}\right) \\
& =-\frac{\Gamma((N+1) / 2) \Gamma(n+1 / 2)}{\sqrt{\pi} \Gamma(n+(N-1) / 2)}\left(1-\frac{u^{2}}{r^{2}}\right)^{(N-1) / 2} \frac{2 u}{r} F\left(1-n, n+\frac{N}{2} ; \frac{3}{2} ; \frac{u^{2}}{r^{2}}\right)
\end{aligned}
$$

(for the relationship between Gegenbauer's polynomials and the Gauss hypergeometric functions see, e.g., [12, formula (8.932)]). Note that the expressions involving Gegenbauer's polynomials can also be obtained by inverting the Fourier transform (3.8) mentioned above. Remaining $k_{0}$ is obtained by integrating (3.7) with respect to $2 \sin (\lambda u) d \lambda$ / $\pi\left|s^{N}\right|$ over $\mathbb{R}_{+}$. Since

$$
\frac{2}{\pi} \int_{0}^{\infty} \frac{1-\cos (\lambda w)}{\lambda} \sin (\lambda u) d \lambda=1_{(u, r)}(w)
$$

(see $[12$, formulas $(3.721 .1)$ and $(3.741 .2)])$, we obtain

$$
\pi\left|s^{N-1}\right| k_{0}(r, u)=(N-1) \int_{u / r}^{1}\left(1-y^{2}\right)^{(N-3) / 2} d y .
$$

Thus Corollary 5.2 yields the following.

Theorem 6.1. Let $X$ be Lévy's Brownian motion on $\mathbb{R}^{N}$. It can be represented as

$$
X(t)=\sqrt{\frac{2}{\pi}} \sum_{l=0}^{\infty} \sum_{m=1}^{h(l, N)} S_{l}^{m}\left(\frac{t}{\|t\|}\right) \int_{0}^{\|t\|} \pi\left|s^{N-1}\right| k_{l}(\|t\|, u) d M_{l}^{m}(u),
$$

where the kernels $\pi\left|s^{N-1}\right| k_{l}$ are given by (6.5)-(6.8), while $\left\{M_{l}^{m}\right\}$ are independent copies of a standard Brownian motion.

Remark 6.2. The kernels (6.5)-(6.8) occurred already in [17], in which McKean Jr. has pointed out that these kernels are in fact singular in the sense that a nontrivial square integrable function can be found that is orthogonal to $k_{l}$ when $l>2$. He has shown how 
to replace them by more convenient nonsingular kernels that admitted him to confirm Lévy's conjecture that the Brownian motions in odd-dimensional spaces are Markov, but not in even-dimensional spaces. Obviously, the transition from singular to nonsingular kernels is highly desirable in the present setting as well; however, this step would require considerable refining of the theory and would bring us too far afield. We intend to return to this subject in our forthcoming work.

In conclusion, we apply Theorem 4.1 to the present case.

Theorem 6.3. Let $X$ be Lévy's Brownian motion on $\mathbb{R}^{N}$. It can be represented on the ball $\mathscr{B}_{T}$ of radius $T$ (cf. (4.1)) as follows:

$$
X(t)=\sum_{n=0}^{\infty} \sum_{l=0}^{\infty} \sum_{m=1}^{h(l, N)} S_{l}^{m}\left(\frac{t}{\|t\|}\right) G_{l}\left(\|t\|, \omega_{n}\right) \xi_{l, n}^{m}, \quad t \in \mathscr{B}_{T},
$$

where

$$
\omega_{n}=\frac{(2 n+1) \pi}{2 T}
$$

and the $\xi_{l, n}^{m}$ are independent mean-zero Gaussian random variables with variances

$$
\sigma_{n}^{2}=\frac{4 \pi^{(N+1) / 2} \Gamma((N+1) / 2)}{T \Gamma^{2}(N / 2)} .
$$

This series converges with probability one in the space of continuous functions on $\mathscr{B}_{T}$.

Remark 6.4. Note that in the scalar case $N=1$ we obtain a series representation of standard Brownian motion on $[0,1]$,

$$
W(t)=\sqrt{2} \sum_{n=0}^{\infty} \frac{1-\cos (t(n+1 / 2) \pi)}{(n+1 / 2) \pi} \xi_{n}^{0}+\sqrt{2} \sum_{n=0}^{\infty} \frac{\sin (t(n+1 / 2) \pi)}{(n+1 / 2) \pi} \xi_{n}^{1},
$$

where $\left\{\xi_{n}^{0}\right\}$ and $\left\{\xi_{n}^{1}\right\}$ are independent sequences of standard Gaussian independent random variables, so that (6.10) can be viewed as a multivariate version of the classical PaleyWiener expansion.

6.2. Lévy's fractional Brownian motion. Lévy's fractional Brownian motion is defined on $\mathbb{R}^{N}$ as a centered Gaussian random field with covariance function

$$
\mathbb{E} X(t) X(s)=\frac{1}{2}\left(\|t\|^{2 H}+\|s\|^{2 H}-\|t-s\|^{2 H}\right),
$$

where $H \in(0,1)$ is called the Hurst index. Observe that for $H=1 / 2$ it reduces to Lévy's Brownian motion considered in the preceding section. In the present case the structural function is $D(r)=r^{2 H}$, so that we can argue like in the previous section to determine the corresponding spectral function. First, formula (3.12) is rewritten in the form (6.2) but with $r^{2 H}$ instead of $r$, and then the density of the form $\lambda^{1+2 H} \Phi^{\prime}(\lambda)=c_{H N}^{2}\left|s^{N-1}\right|$ is sought, 
with a constant $c_{H N}^{2}$ to be determined by [12, formula (6.561.14)]. By straightforward calculations we arrive at

$$
c_{H N}^{2}=\frac{\Gamma(H+N / 2) \Gamma(1+H) \sin (\pi H)}{\pi^{(N+2) / 2} 2^{1-2 H}} .
$$

Thus by (3.18) we deal here with the spectral measure

$$
\mu(d \lambda)=c_{H N}^{2} \lambda^{1-2 H} d \lambda
$$

that differs only by a constant factor from the spectral measure in [8, Section 4]. Therefore, the expressions for the mass function $\mathbf{m}$ and the eigenfunctions $A$ and $B$, obtained in the aforementioned work, can be easily adapted to the present situation with the help of "rule 1 " of [6, page 265]. We get

$$
\begin{gathered}
\mathbf{m}(x)=\frac{\kappa_{H N}^{1 / H}}{4 H(1-H)} x^{(1-H) / H} \\
A(x, \lambda)=\Gamma(1-H)\left(\frac{\lambda}{2}\right)^{H} \sqrt{\kappa_{H N} x} J_{-H}\left(\lambda\left(\kappa_{H N} x\right)^{1 / 2 H}\right), \\
B(x, \lambda)=\frac{\kappa_{H N} \Gamma(1-H)}{2 H}\left(\frac{\lambda}{2}\right)^{H}\left(\kappa_{H N} x\right)^{(1-H) / 2 H} J_{1-H}\left(\lambda\left(\kappa_{H N} x\right)^{1 / 2 H}\right) .
\end{gathered}
$$

The new constant is

$$
\kappa_{H N}=\frac{2 \pi^{(N+2) / 2}}{\Gamma(H+N / 2) \Gamma(1-H)}
$$

(it in fact extends the constant $\kappa_{H 1}$ appearing in [8, Section 4], to the multidimensional case). After the necessary substitution $x(t)=t^{2 H} / \kappa_{H N}$ this constant does not occur in the eigenfunctions

$$
\begin{gathered}
A(x(t), \lambda)=\Gamma(1-H)\left(\frac{\lambda t}{2}\right)^{H} J_{-H}(\lambda t), \\
B(x(t), \lambda) x^{\prime}(t)=\Gamma(1-H)\left(\frac{\lambda t}{2}\right)^{H} J_{1-H}(\lambda t),
\end{gathered}
$$

but it does enter in the expression of the variance function

$$
V(2 t)=\frac{m(x(t))}{\pi}=\frac{\kappa_{H N} t^{2-2 H}}{4 H(1-H) \pi}=\frac{\pi^{N / 2} t^{2-2 H}}{2 H \Gamma(2-H) \Gamma(H+N / 2)} .
$$

The assertion of Theorem 6.1 is extended to the present fractional case as follows.

Theorem 6.5. Let $X$ be Lévy's fractional Brownian motion on $\mathbb{R}^{N}$ with Hurst index $H \in$ $(0,1)$. Then it is represented as follows:

$$
X(t)=\sqrt{2}\left|s^{N-1}\right| \sum_{l=0}^{\infty} \sum_{m=1}^{h(l, N)} S_{l}^{m}\left(\frac{t}{\|t\|}\right) \int_{0}^{\|t\|} \pi k_{l}(\|t\|, u) d M_{l}^{m}(u),
$$


where $\left\{M_{l}^{m}\right\}$ are independent copies of a Gaussian martingale $M$ with mean zero and the variance function $\mathbb{E}|M(u)|^{2}=V(2 u)$ given by (6.21), with the kernels $k_{l}$ defined by

$$
\frac{\pi k_{0}(r, u)}{c_{H N}^{2} \Gamma(N / 2)}=\frac{2 \Gamma(1-H)}{\Gamma(H-1+N / 2)}\left(\frac{2}{u}\right)^{1-2 H} \int_{u / r}^{1} y^{1-2 H}\left(1-y^{2}\right)^{H-2+N / 2} d y,
$$

for $n=0,1,2, \ldots$,

$$
\begin{aligned}
\frac{\pi k_{2 n+1}(r, u)}{c_{H N}^{2} \Gamma(N / 2)}= & -\frac{\Gamma(n+1-H)}{\Gamma(n+H+N / 2)}\left(\frac{2}{r}\right)^{1-2 H}\left(1-\frac{u^{2}}{r^{2}}\right)^{H-1+N / 2} \\
& \times F\left(-n, n+\frac{N}{2} ; 1-H ; \frac{u^{2}}{r^{2}}\right)
\end{aligned}
$$

and for $n=1,2, \ldots$,

$$
\begin{aligned}
\frac{\pi k_{2 n}(r, u)}{c_{H N}^{2} \Gamma(N / 2)}= & -\frac{\Gamma(n+1-H)}{(1-H) \Gamma(n+H-1+N / 2)}\left(\frac{2}{r}\right)^{1-2 H}\left(1-\frac{u^{2}}{r^{2}}\right)^{H-1+N / 2} \\
& \times \frac{u}{r} F\left(1-n, n+\frac{N}{2} ; 2-H ; \frac{u^{2}}{r^{2}}\right) .
\end{aligned}
$$

Proof. We need the inverse transforms of function $G_{l}$ with respect to the measure (6.16), as defined by formula (3.20). Since the eigenfunctions are given by (6.19) and (6.20), it follows from (5.2) that for $l>0$ the kernels $k_{l}$ are evaluated as Hankel transforms of the following form:

$$
\begin{aligned}
k_{2 n+1}(r, u) & =\check{G}_{2 n+1}(r, x(u)) \\
& =\frac{2^{1-H} c_{H N}^{2} \Gamma(1-H) u^{H}}{\pi} \int_{0}^{\infty} G_{2 n+1}(r, \lambda) J_{-H}(u \lambda) \lambda^{1-H} d \lambda, \\
k_{2 n}(r, u) & =\check{G}_{2 n}(r, x(u)) x^{\prime}(u) \\
& =\frac{2^{1-H} c_{H N}^{2} \Gamma(1-H) u^{H}}{\pi} \int_{0}^{\infty} G_{2 n}(r, \lambda) J_{1-H}(u \lambda) \lambda^{1-H} d \lambda .
\end{aligned}
$$

The required results are then found in the tables in [11, formula (8.11.9)]. To complete the proof we will show

$$
k_{0}(r, u)=\frac{c_{H N}^{2} \Gamma^{2}(1-H)}{\pi}\left(\frac{u}{2}\right)^{2 H-1}\left(1-\frac{B_{u^{2} / r^{2}}(1-H, H-1+N / 2)}{B(1-H, H-1+N / 2)}\right)
$$

where $B_{x}(\alpha, \beta)$ is the incomplete beta function (see [12, formula (8.391)]). Indeed, the kernel $k_{0}$ is computed as the sum of the following two terms. The first term is

$$
c_{H N}^{2} \Gamma(1-H)\left(\frac{u}{2}\right)^{H} \frac{2}{\pi} \int_{0}^{\infty} J_{1-H}(u \lambda) \frac{d \lambda}{\lambda^{H}}=\frac{c_{H N}^{2}}{\pi} \Gamma^{2}(1-H)\left(\frac{u}{2}\right)^{2 H-1}
$$


(the integral is taken by means of [12, formula (6.561.14)]). The second term has the same expression as $k_{2 n}$ given above, but evaluated at $n=0$ (for the relationship between the incomplete Beta function and the Gauss hyperbolic function, see [12, formula (8.391)]).

Remark 6.6. It can be shown in the present fractional case too that the kernels $k_{l}$ with $l>2$ are singular in the same sense as in the special case $H=1 / 2$ already mentioned in Remark 6.2. To see this, observe first that the Gauss hypergeometric functions that occur in the expressions for $k_{l}$ are classical orthogonal polynomials, known in the literature as generalized Gegenbauer polynomials (see, e.g., [5, Section 1.5.2]). It is then straightforward to follow McKean's Jr. arguments in [17]; however, we do not dwell upon this here and note only that the analogue of McKean's Jr. nonsingular kernels to the fractional case is known for the general Hurst index $H$ as well, see Malyarenko [16].

In conclusion we specify our general series expansion of Theorem 4.1 to Lévy's fractional Brownian motion.

Theorem 6.7. Let $\omega_{0}<\omega_{1}<\omega_{2}<\cdots$ be the nonnegative real-valued zeros of the Bessel function $J_{-H}$. Then Lévy's fractional Brownian motion $X$ with Hurst index $H$ restricted to the ball $\mathscr{B}_{T}$ of radius $T$ (cf. (4.1)) can be represented as follows:

$$
X(t)=\sum_{l=0}^{\infty} \sum_{m=1}^{h(l, N)} \sum_{n=0}^{\infty} S_{l}^{m}\left(\frac{t}{\|t\|}\right) G_{l}\left(\|t\|, \frac{\omega_{n}}{T}\right) \xi_{l, n}^{m}, \quad t \in \mathscr{B}_{T},
$$

where the $\xi_{l, n}^{m}$ are independent mean-zero Gaussian random variables with variances

$$
\sigma_{n}^{2}=\frac{2 H \Gamma(H+N / 2)\left|s^{N-1}\right|^{2}}{\pi^{N / 2} T^{2-2 H} \Gamma(1-H)\left(\omega_{n} / 2\right)^{2 H} J_{1-H}^{2}\left(\omega_{n}\right)} .
$$

This series converges with probability 1 in the space of continuous functions on $\mathscr{B}_{T}$.

Proof. By (6.19) we have $A(x(t), \lambda)=0$ if and only if $\lambda=\omega_{n} / T$ and

$$
\left.\frac{\partial}{\partial \omega} A(x(T), \omega)\right|_{\omega=\omega_{n} / T}=-\Gamma(1-H) T\left(\frac{\omega_{n}}{2}\right)^{H} J_{1-H}\left(\omega_{n}\right) .
$$

By $(6.20)$

$$
B\left(x(T), \omega_{n}\right)=\frac{\Gamma(1-H)\left(\omega_{n} / 2\right)^{H} J_{1-H}\left(\omega_{n}\right) \kappa_{H N}}{2 H T^{2 H-1}} .
$$

The required expression for $\sigma_{n}^{2}$ is now verified by (4.9) and (6.21). The assertion of the present theorem thus follows from Theorem 4.1 .

\section{References}

[1] G. E. Andrews, R. Askey, and R. Roy, Special Functions, Encyclopedia of Mathematics and Its Applications, vol. 71, Cambridge University Press, Cambridge, 1999.

[2] R. P. Boas Jr., Entire Functions, Academic Press, New York, 1954. 
[3] N. N. Chentsov, Brownian motion for several parameters and generalized white noise, Theory of Probability \& Its Applications 2 (1957), no. 2, 265-266.

[4] L. de Branges, Hilbert Spaces of Entire Functions, Prentice-Hall, New Jersey, 1968.

[5] C. F. Dunkl and Y. Xu, Orthogonal Polynomials of Several Variables, Encyclopedia of Mathematics and Its Applications, vol. 81, Cambridge University Press, Cambridge, 2001.

[6] H. Dym and H. P. McKean, Gaussian Processes, Function Theory, and the Inverse Spectral Problem, Academic Press, New York, 1976.

[7] K. Dzhaparidze and H. van Zanten, A series expansion of fractional Brownian motion, Probability Theory and Related Fields 130 (2004), no. 1, 39-55.

[8] K. Dzhaparidze, H. van Zanten, and P. Zareba, Representations of fractional Brownian motion using vibrating strings, Stochastic Processes and Their Applications 115 (2005), no. 12, 19281953.

[9] A. Erdélyi, W. Magnus, F. Oberhettinger, and F. G. Tricomi, Higher Transcendental Functions. Vol. II, McGraw-Hill, New York, 1953.

[10] _ Tables of Integral Transforms. Vol. I, McGraw-Hill, New York, 1954.

[11] _ Tables of Integral Transforms. Vol. II, McGraw-Hill, New York, 1954.

[12] I. S. Gradshteyn and I. M. Ryzhik, Table of Integrals, Series, and Products, Academic Press, New York, 1980.

[13] S. Kac and M. G. Krein, On the spectral functions of the string, American Mathematical Society Translations 103 (1974), 19-102.

[14] N. Leonenko, Limit Theorems for Random Fields with Singular Spectrum, Mathematics and Its Applications, vol. 465, Kluwer Academic, Dordrecht, 1999.

[15] P. Lévy, Processus Stochastiques et Mouvement Brownien. Suivi d'une note de M. Loève, GauthierVillars, Paris, 1948.

[16] A. Malyarenko, A series expansion of a certain class of isotropic Gaussian random fields with homogeneous increments, preprint, 2005.

[17] H. P. McKean Jr., Brownian motion with a several-dimensional time, Theory of Probability and Its Applications 8 (1963), 357-378.

[18] G. M. Molchan, On some problems concerning Brownian motion in Lévy's sense, Theory of Probability and Its Applications 12 (1967), no. 4, 682-695.

[19] A. W. van der Vaart and J. A. Wellner, Weak Convergence and Empirical Processes, Springer Series in Statistics, Springer, New York, 1996.

[20] N. J. Vilenkin, Special Functions and the Theory of Group Representations, Translated from the Russian by V. N. Singh. Translations of Mathematical Monographs, Vol. 22, American Mathematical Society, Rhode Island, 1968.

[21] A. M. Yaglom, Correlation Theory of Stationary and Related Random Functions. Vol. II, Springer Series in Statistics, Springer, New York, 1987.

Kacha Dzhaparidze: Department PNA, Center for Mathematics and Computer Science, Kruislaan 413, Amsterdam 1098 SJ, The Netherlands

E-mail address: kacha@cwi.nl

Harry van Zanten: Department of Mathematics, Vrije Universiteit Amsterdam, De Boelelaan 1081a, Amsterdam $1081 \mathrm{HV}$, The Netherlands

E-mail address: harry@cs.vu.nl

Pawel Zareba: Department of Mathematics, Vrije Universiteit Amsterdam, De Boelelaan 1081a, Amsterdam $1081 \mathrm{HV}$, The Netherlands

E-mail address: pzareba@few.vu.nl 


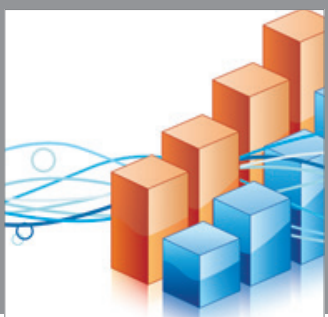

Advances in

Operations Research



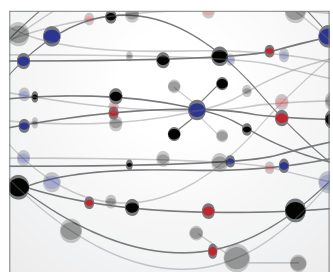

\section{The Scientific} World Journal


International Journal of

Mathematics and

Mathematical

Sciences


Journal of

Applied Mathematics
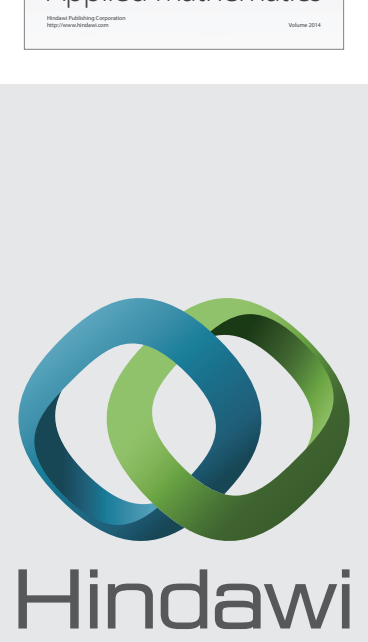

Submit your manuscripts at http://www.hindawi.com
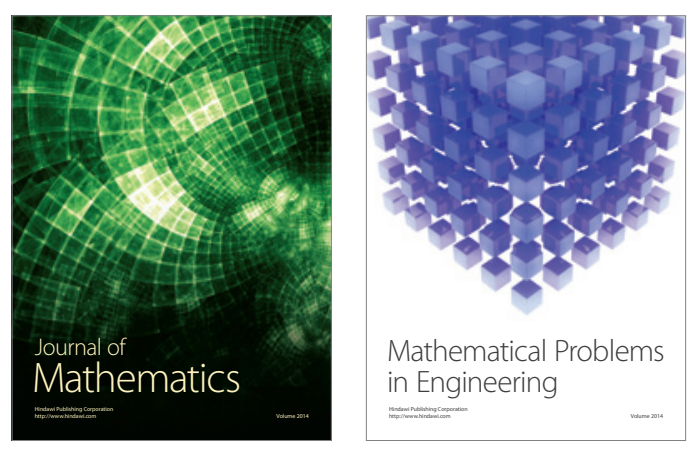

Mathematical Problems in Engineering
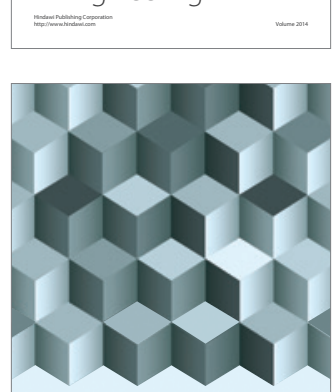

Journal of

Function Spaces
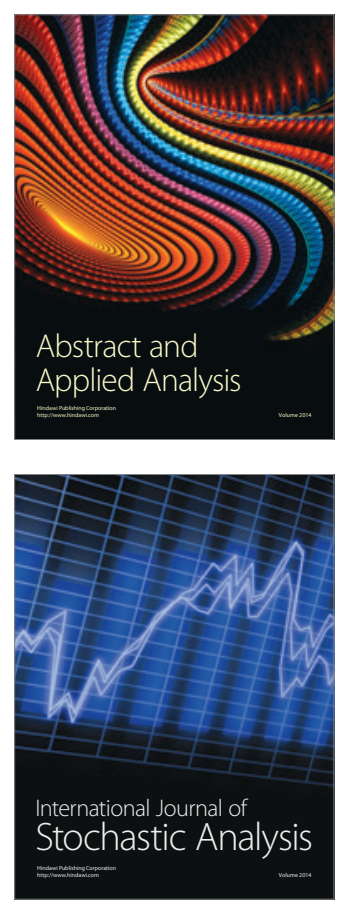

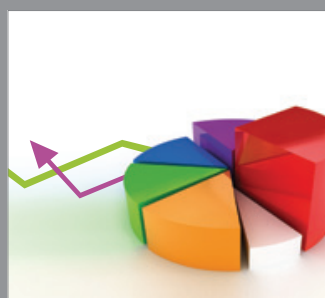

ournal of

Probability and Statistics

Promensencen
